\title{
A Hugoniot theory for solid and powder mixtures
}

\author{
Barry R. Krueger and Thad Vreeland, Jr. \\ Keck Laboratory of Engineering Materials, California Institute of Technology, 138-78, Pasadena, \\ California 91125
}

(Received 11 May 1990; accepted for publication 19 September 1990)

\begin{abstract}
A model is presented from which one can calculate the Hugoniot of solid and porous twocomponent mixtures up to moderate pressures using only static thermodynamic properties of the components. The model does not presuppose either the relative magnitude of the thermal and elastic energies or temperature equilibrium between the two components. It is shown that for a mixture, the conservation equations define a Hugoniot surface and that the ratio of the thermal energy of the components determines where the shocked state of the mixture lies on this surface. This ratio, which may strongly affect shock-initiated chemical reactions and the properties of consolidated powder mixtures, is found to have only a minor effect on the Hugoniot of a mixture. It is also found that the Hugoniot of solids and solid mixtures is sensitive to the pressure derivative of the isentropic bulk modulus at constant entropy.
\end{abstract}

\section{INTRODUCTION}

The Hugoniot of a mixture is intimately related to the current interest in shock-initiated chemical reactions. ${ }^{1-5}$ With the high temperature and pressure associated with shock wave processing, it may be possible to concurrently synthesize and form near-net-shape parts of intermetallic compounds and other materials. Shock processing is also a viable technology for producing composite materials where it is necessary to control chemical reactions between the matrix and reinforcing powders since such reactions often have deleterious effects on the mechanical properties of the composite.

Fully understanding shock-initiated chemical reactions and shock compaction of composites is dependent upon knowing the Hugoniot of the mixture of interest. To this end, several models have been put forth. A popular approach has been developed by McQueen et al. ${ }^{6}$ Their theory requires the Hugoniots of the components and assumes the thermal energy of a shocked mixture to be small compared to the elastic energy. This assumption is necessary since their model does not account for a difference in the temperature rise of the components which will occur in shocked solid, and particularly powder, mixtures. Duvall and Taylor ${ }^{7}$ have used a mixture method that relies on knowing the component's Gibb's free energy, and they assume the components to be in thermal equilibrium.

Both of these approaches require data that is often sparse or tedious to determine. Furthermore, in shocked powders the relative magnitude of the thermal and compressional energy is just the opposite of the assumption of McQueen et al. The difference in temperature of the two components of a mixture may be large ${ }^{8}$ and, in most materials, will not equilibrate quickly. ${ }^{7}$ In light of this, we have developed a model which allows for large thermal energies and does not require either thermal equilibrium or the $\mathrm{Hu}$ goniots of the component materials.

\section{PRELIMINARY DISCUSSION}

For the proposed Hugoniot theory of a two component mixture, two preliminary assumptions are made: (i) the components are at equal pressures; (ii) the components have equal particle velocities. Assumption (i) is justified as follows. If the pressures were initially different, equilibration would occur within a few multiples of a time $t-d / c_{0}$, where $d$ is an average particle diameter and $c_{0}$ is some average speed of sound. ${ }^{7}$ For typical materials, $c_{0}$ is on the order of $5 \times 10^{3} \mathrm{~m} / \mathrm{s}$, and for powders and some multiphase materials, typical component diameters are on the order of 100 $\mu \mathrm{m}$ giving a characteristic pressure equilibration time of approximately $100 \mathrm{~ns}$. This is on the order of the shock rise time in ductile powders ${ }^{\circ}$ indicating that pressure equilibration will occur during the shock rise time or within tens of nanoseconds thereafter. In systems subject to chemical reactions, it has been shown that the reactions may initiate within the shock front in ultrafine powders. ${ }^{10}$ If extensive reactions do occur within $100 \mathrm{~ns}$ or less, then the present model is not applicable.

The second assumption, that the components have equal particle velocities behind the shock, is based on experimental evidence. In shock compaction experiments on 1:1at. $\% \mathrm{Ni} / \mathrm{Si}, \mathrm{Ni} / \mathrm{Ti}$, and $\mathrm{Ni} / \mathrm{Cu}$ powders, we see no evidence that the components maintain different particle velocities. If this were true, the lower shock impedance material would segregate in the shock direction which is not observed. There is also no known experimental evidence in the literature that the two components of a mixture maintain different particle velocities, although differences in particle velocities have been used to explain some shock-initiated chemical reactions. $^{3}$

Assumptions (i) and (ii), together with conservation of mass, momentum, and energy, imply that the two components absorb different amounts of energy and are therefore, in general, at different average temperatures immediately 
behind the shock front. An example supporting the implied energy partitioning is the theoretical and experimental work on the shock consolidation of $\mathrm{Al} / \mathrm{SiC}$ metal matrix composites from $\mathrm{Al}$ and $\mathrm{SiC}$ rods, (i.e., two-dimensional powders).${ }^{8}$ Further evidence is shown in Fig. 1 which is a micrograph of a shock-consolidated mixture of hard and soft maraging steel powders heat treated to VH 620 and 280, respectively. The softer, light-etching particles have deformed significantly in comparison with the harder particles. Intuitively, in a mixture of soft and hard, small and large, or irregular and regularly shaped particles, one would expect the former to absorb more energy than the latter which will result in different average particle temperatures behind the shock.

A simple argument reveals that for typical powders, a temperature difference will not equilibrate quickly, but in fact, orders of magnitude more slowly than any pressure difference. Significant thermal conduction will occur over distances $d=\sqrt{\kappa t}$, where $\kappa$ is some average thermal diffusivity. Using typical parameters of $d=100 \mu \mathrm{m}$ and the thermal diffusivity of a good thermal conductor such as $\mathrm{Cu}$ indicates that temperature differences will equilibrate 2 to 3 orders of magnitude more slowly than pressure differences and in a time which may be longer than the shock duration itself. ${ }^{7}$ Obviously, the temperature must be continuous across the particle boundaries of the two components, but the proposed theory is a bulk thermodynamic model which considers average component temperatures.

If the particle size is approximately $100 \mathrm{~nm}$ or smaller, the difference between pressure and temperature equilibration times becomes small, and the equilibration times are on the order of the shock rise time. This effect has been exploited by Boslough in his measurements of shock temperatures in thermite and other systems using radiation pyro-

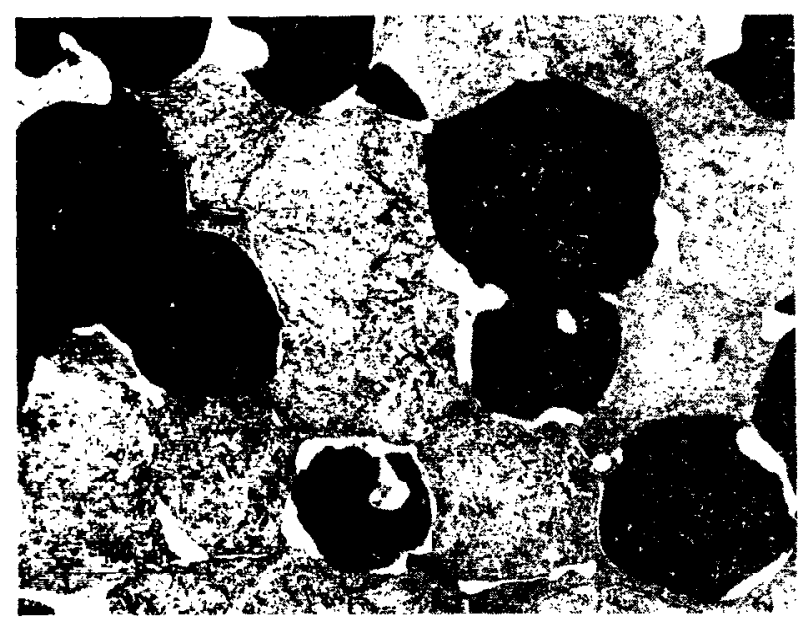

FIG. 1. Shock-consolidated $-100+200$ mesh maraging steel powders. The dark and light particles had a preshocked vickers microhardness of 280 and 640 , respectively. The initial porosity was $32.0 \%$. The $50 / 50$ hard-tosoft mixture was impacted by a 304 stainless steel flyer at $986 \mathrm{~m} / \mathrm{s}$. The soft particles deformed significantly compared to the hard particles as can be seen by the concavity of the interfaces. The very light material at the interfaces is rapidly quenched amorphous material. metry. ${ }^{10,11}$ The theory presented here can treat ultrafine particle shock consolidation by assuming a thermal energy ratio such that the components are at equal temperatures as will be explained below.

\section{THEORY}

With the assumptions discussed above, the laws of conservation of mass, momentum, and energy will be no different for a mixture provided that no chemical reactions occur: conservation of mass:

$$
\rho_{00 A B} C_{S}=\rho_{1 A B}\left(C_{S}-u_{1}\right)
$$

conservation of momentum:

$$
P_{1}-P_{0}=\rho_{0 \cap A B} C_{S} u_{1}=\rho_{1 A B}\left(C_{S}-u_{1}\right) u_{1},
$$

conservation of energy:

$$
\rho_{00 A B} C_{S}\left(E_{1}-E_{0}+\frac{1}{2} u_{1}^{2}\right)=P_{1} u_{1},
$$

where the $\rho_{00 A B}$ is the initial density of the mixture; $\rho_{1 A B}$ is the shocked density; $C_{S}$ is the shock velocity; $u_{1}$ is the particle velocity; $P_{0}$ is the initial pressure; $P_{1}$ is the shock pressure, and $E_{0}$ and $E_{1}$ are the initial and final specific internal energy of the mixture, respectively. Substituting Eqs. (1) and (2) into Eq. (3) and dividing the specific internal energy between the two components gives

$$
x \Delta E_{A}+(1-x) \Delta E_{B}=\frac{1}{2} P_{1}\left(V_{00 A B}-V_{1 A B}\right),
$$

where $x$ is the mass fraction of material $A ; V_{0 O A B}$ and $V_{1 A B}$ are the initial and shocked specific volumes of the mixture, respectively. $E_{A}$ and $E_{B}$ are the specific internal energy of the two components, and the initial pressure is assumed to be zero. Equation (4) is a simple expansion of the familiar equation $E=\frac{1}{2} P\left(V_{00}-V_{1}\right)$. The $\Delta$ 's can be removed if the ambient energy is used as a reference.

The energy and pressure of each constituent can be separated into thermal and elastic (isentropic) components,

$$
\begin{aligned}
& E_{A}=E_{T A}+E_{E A}, \quad E_{B}=E_{T B}+E_{E B}, \\
& P_{A}=P_{T A}+P_{E A}=P_{B}=P_{T B}+P_{E B},
\end{aligned}
$$

where the $E$ and $T$ subscripts refer to the elastic and the thermal components, respectively. The second equality in $\mathrm{Eq}$. (6) is due to the equal pressure assumption. Using the definition of the Grüneisen parameter $\gamma \equiv V(\partial P / \partial E)_{v}$ and assuming each component's Grüneisen parameter-to-volume ratio is constant and temperature independent, the thermal pressure in terms of the thermal energy of the two components becomes

$$
\begin{aligned}
& d P_{T A}=\frac{\gamma_{A} d E_{T A}}{V_{A}} \approx \frac{\gamma_{0 A} d E_{T A}}{V_{O A}} \Rightarrow P_{T A}=\frac{\gamma_{0 A} E_{T A}}{V_{0 A}}, \\
& d P_{T B}=\frac{\gamma_{B} d E_{T B}}{V_{B}} \approx \frac{\gamma_{0 B} d E_{T B}}{V_{O B}} \Rightarrow P_{T B}=\frac{\gamma_{O B} E_{T B}}{V_{O B}} .
\end{aligned}
$$

Equations (5)-(7) can be substituted into Eq. (4) yielding 
$P_{1}=\frac{\sigma_{A} P_{E A}\left(\lambda_{A}\right)+\sigma_{B} P_{E B}\left(\lambda_{B}\right)-\frac{x E_{E A}\left(\lambda_{A}\right)}{V_{0 A} V_{O B}}-\frac{(1-x) E_{E B}\left(\lambda_{B}\right)}{V_{0 A} V_{0 B}}}{\sigma_{A}+\sigma_{B}-\frac{1}{2}\left(\varphi-\eta_{A} \lambda_{A}-\eta_{B} \lambda_{B}\right)}$,

where

$$
\begin{aligned}
& \sigma_{A}=\frac{x}{\gamma_{0 A} V_{0 B}}, \quad \sigma_{B}=\frac{(1-x)}{\gamma_{0 B} V_{0 A}}, \\
& \eta_{A}=\frac{x}{V_{O B}}, \quad \eta_{B}=\frac{(1-x)}{V_{0 A}}, \\
& \lambda_{A}=\frac{V_{1 A}}{V_{0 A}}, \quad \lambda_{B}=\frac{V_{1 B}}{V_{0 B}}, \quad \varphi=\frac{V_{0 O A B}}{V_{0 A} V_{0 B}} .
\end{aligned}
$$

$V_{1 A}$ and $V_{1 B}$ are the shocked specific volumes of the $A$ and $B$ components, respectively. The volume dependence $\left(\lambda_{i}\right)$ of the elastic pressures and energies is indicated, and hydrodynamic material behavior is assumed. The distension of the powder, $m \equiv V_{O A A B} / V_{O A B}$ where $V_{0 A B}$ is the solid volume of the mixture at standard conditions, enters Eq. (8) through the parameter $\varphi$.

Next, one must choose expressions for the elastic pressures and energies. By assuming a linear dependence of the isothermal bulk modulus with pressure, Murnaghan ${ }^{12}$ derived the equation

$P_{E}=\frac{\beta_{0 T}}{\beta_{0 T}^{\prime}}\left[\left(\frac{V_{0}}{V_{1}}\right)^{\beta_{i r}}-1\right]=\frac{\beta_{O T}}{\beta_{0 T}^{\prime}}\left[\lambda^{-\beta_{i r}^{\prime}}-1\right]$

where $\beta_{0 T}$ is the isothermal bulk modulus at standard conditions, and $\beta_{O T}^{\prime}$ is its first derivative with respect to pressure at constant temperature. Anderson ${ }^{13}$ has shown that Eq. (9) is a good approximation over a wide range of materials and to volume ratios of around 0.8 . However, the elastic pressure in the shock process is not isothermal but rather isentropic. Integrating at constant entropy, a linear relationship between the isentropic bulk modulus and pressure yields a similar equation,

$$
P_{E}=\frac{\beta_{0 S}}{\beta_{0 S}^{\prime}}\left[\left(\frac{V_{0}}{V_{1}}\right)^{\beta_{i s}^{\prime}}-1\right]=\frac{\beta_{0 S}}{\beta_{0 S}^{\prime}}\left[\lambda^{-\beta_{0 S S}^{\prime}}-1\right],
$$

where $\beta_{0 S}$ is the isentropic bulk modulus at standard conditions, and $\beta{ }_{0 S}^{\prime}$ is its first derivative with respect to pressure at constant entropy. Then, Eq. (10) can be integrated to get the elastic energy. The difficulty with Eq. (10) is that $\beta_{0 s}^{\prime}$ has not been measured experimentally nor can it be derived easily from measurable thermodynamic parameters. Initially, it has been assumed that $\left(\partial \beta_{S} / \partial P\right)_{S} \equiv \beta_{S}^{\prime} \approx\left(\partial \beta_{S} / \partial P\right)_{T}$ which has been determined for some substances using static pressure sound velocity measurements. ${ }^{13}$ If these measurements are not available, it has been assumed that $\beta_{S}^{\prime} \approx\left(\partial \beta_{T} / \partial P\right)_{T}$, and $\left(\partial \beta_{T} / \partial P\right)_{T}$ was calculated from equation of state data in Ref. 14 with the exception of A12024 where the Dugdale-MacDonald relationship ${ }^{15}$ was used, $\left(\partial \beta_{T} / \partial P\right)_{T}=2 \gamma+1$. According to Anderson's data, the value of the two approximations for $\beta_{S}^{\prime}$ do not differ greatly. Their validity will be discussed later.

Note that Eq. (8) is valid for all porosities since no as- sumptions were made in its derivation concerning the relative magnitude of the thermal and elastic energy components except that the ratio $\gamma / V$ is constant and independent of temperature. $\mathrm{Oh}^{16}$ has shown that the constant $\gamma / V$ approximation is inaccurate at very high energies, however this discrepancy has been allowed since most shock compaction and shock-initiated reaction experiments are conducted at moderate energies.

Equation (8) gives the shock pressure in terms of the two component's volumes. For a single material, the shocked volume is determined as a function of pressure. Then, this equation together with the equation $u_{1}=\sqrt{P \Delta V}$ and a known flyer pressure-particle velocity relationship can be used to determine all the shock parameters. However since Eq. (8) gives the pressure in terms of both volumes, there is one more unknown parameter. In other words, Eq. (8) is a Hugoniot surface that depends on the individual volumes of the components rather than simply the total shocked volume.

Since there exists one more unknown, we initially considered measuring one more shock parameter, specifically the shock velocity since it lends itself easily to measurement. With a known shock velocity, Eqs. (1), (2), and (8) together with Eq. (9) and a known flyer pressure-particle velocity relationship constitute a system of four equations with four unknowns: $P_{1}, u_{1}, V_{1 A}$, and $V_{1 B}$. Once the unknowns are determined, Eqs. (6), (7), and (10) can be used to determine the thermal energy of the two components and hence their temperatures.

Calculations show that small variations (a few percent) of the shock speed away from the value calculated assuming averaged properties may lead to nonphysical results such as a negative thermal energy for one of the components. Therefore, the theory predicts that the shock velocity in a mixture is near the shock velocity assuming averaged properties, and that any difference will probably be smaller than the resolution of a shock speed measurement. Another approach is to assume the shock speed is the value calculated using averaged properties. This results in calculated energy partitioning that is nonintuitive and contradicts experiments in certain powder mixtures such as TiAl-6V-4 and SiC where the very hard $\mathrm{SiC}$ deforms relatively little while the Hugoniot assuming a shock velocity calculated from averaged properties predicts that it absorbs significant thermal energy.

A third approach is to obtain a fifth equation by recognizing that at given shock conditions there exists a thermal energy partitioning ratio that is determined by the relative mechanical properties, sizes, and shapes of the two components. The softer, smaller, and irregularly shaped component absorbs more thermal energy, or if one wished to assume equal temperatures, as in the case of ultrafine powders, it is possible to determine an approximate thermal energy ratio based on the specific heats of the components over 
some expected temperature range. Except for the last case, quantitatively predicting the thermal energy partitioning for a given mixture is difficult. Nevertheless, at given shock conditions, there does exist a thermal energy ratio of the form

$$
\xi\left(P_{1}, C_{S}, u_{1}\right) \equiv E_{T B} / E_{T A} .
$$

Using Eqs. (6), (7), and (11), we obtain the equation

$$
P_{1}(1-\xi / \epsilon)=P_{E B}\left(\lambda_{B}\right)-(\xi / \epsilon) P_{E A}\left(\lambda_{A}\right),
$$

where $\epsilon=V_{O B} \gamma_{O A} / V_{O A} \gamma_{0 B}$. Equations (1), (2), (8), and (12) and a known flyer pressure-particlc velocity relationship constitute a system of five equations and five unknowns, $C_{S}, P_{1}, u_{1}, V_{1 A}$, and $V_{1 B}$, which can be solved numerically.

To determine the effects of thermal energy partitioning on a mixture's Hugoniot, we have assumed the simplest possible form for Eq. (11), $\xi=$ constant. Doing so simplifies the calculations, but more important, assuming $\xi$ is constant includes the extreme possibility that one component absorbs no thermal energy (i.e., $\xi=0$ or $\infty$ ).

\section{VALIDATION OF THE MODEL FOR HOMOGENEOUS MATERIALS}

To test the madel, the Hugoniots of solid single-component materials were calculated. As discussed earlier, this is the degenerate case of a two-component mixture, and defining a thermal energy ratio is not necessary. The materials were chosen based on the availability of thermodynamic data to approximate $\beta_{S}^{\prime}$ and the availability of statistically significant Hugoniot data over a range of compression where a first-order thermal expansion of a material's isentropic bulk modulus is expected to be valid. The materials and the thermodynamic data used are shown in Table I. Note that most of the materials are metals. Unfortunately, either the thermodynamic or Hugoniot data is not available to compare calculated and experimental results for nonmetallic materials other than $\mathrm{NaCl}$.

A result of the calculations is that the $C_{S}-u_{1}$ relationship is nearly linear as is found experimentally. For all the calculated single-component Hugoniots, the correlation coeffi-

TABLE I. Materials and thermodynamic data used to calculate single-material Hugoniots.

\begin{tabular}{rrrrr}
\hline & \multicolumn{1}{c}{$\begin{array}{c}\text { Density } \\
\text { Material }\end{array}$} & $\begin{array}{c}\beta_{s} \\
\left(\mathrm{~g} / \mathrm{cm}^{3}\right)\end{array}$ & $\beta_{s}^{\prime}$ (Est.) & $\gamma_{0}$ \\
\hline $\mathrm{Cu}$ & 8.94 & 139.76 & 4.994 & 2.04 \\
$\mathrm{Zn}$ & 7.14 & 65.40 & 5.421 & 2.38 \\
$\mathrm{Nb}$ & 8.60 & 175.40 & 3.551 & 1.69 \\
$\mathrm{Au}$ & 19.24 & 179.50 & 5.270 & 3.05 \\
$\mathrm{Pd}$ & 11.95 & 189.00 & 5.655 & 2.18 \\
$\mathrm{Co}$ & 8.82 & 194.60 & 4.700 & 1.99 \\
$\mathrm{~W}$ & 19.20 & 308.10 & 3.996 & 1.54 \\
$\mathrm{Cd}$ & 8.64 & 48.57 & 7.015 & 2.20 \\
$\mathrm{NaCl}$ & 2.16 & 24.70 & 5.270 & 1.57 \\
$\mathrm{Mg}$ & 1.74 & 35.58 & 4.050 & 1.50 \\
$\mathrm{Ag}$ & 10.50 & 108.70 & 5.660 & 2.46 \\
$\mathrm{Al}-2024$ & 2.79 & 79.21 & 5.000 & 2.00 \\
$\mathrm{Ni}$ & 8.90 & 192.50 & 4.620 & 1.91 \\
$\mathrm{~Pb}$ & 11.34 & 46.36 & 4.350 & 2.78 \\
\hline \hline
\end{tabular}

cient between $C_{S}$ and $u_{1}$ is greater than 0.995 . Therefore, the proposed model qualitatively fits the experimental Hugoniot data. The results are shown in Table II in the form $C_{S}=A+B^{*} u_{1}$ along with the values of $A$ and $B$ determined from a linear regression fit of the experimental data ${ }^{17}$ over the velocity ranges indicated. As can be seen in Table II, the calculated shock intercepts match the experimental values well. The average absolute difference between the calculated and experimental values is only $59 \mathrm{~m} / \mathrm{s}$. However, the calculated particle velocity coefficients are consistently higher than the experimental values with an average difference of $14.4 \%$.

By varying the thermodynamic parameters within a reasonable range of uncertainty, it was determined that the calculated particle velocity coefficients are sensitive to $\beta_{S}^{\prime}$, which is the only input parameter that is estimated, while varying $\beta_{S}^{\prime}$ has litle effect on the shock intercept. This indicates that $\beta_{S}^{\prime}$ has an important effect on the slope of a solids Hugoniot in the $C_{S^{-}} u_{1}$ plane and that further theoretical and experimental work is needed in determining its value.

The Hugoniots of the solids in Table I were recalculated with the same parameters except for $\beta$ ', which were adjusted so the calculated particle velocity coefficients fit the experimental values. Table III shows the values of $\beta_{s}^{\prime}$ necessary to fit the experimental particle velocity coefficients and the ratio of the fitted value to the original estimate. This ratio lies between 0.69 and 0.91 and roughly varies inversely with the material's bulk modulus. Also shown are the new values of the calculated shock intercept which differ little from the values calculated originally and the experimental valucs.

These comparisons of calculated and experimental data show that the model qualitatively fits experimental solid $\mathrm{Hu}-$ goniots using estimates of $\beta_{s}^{\prime}$ and that the values of $\beta_{s}^{\prime}$ can be varied so that the calculated solid Hugoniots fit the experimental data. Since actual values of $\beta_{S}^{\prime}$ have not been determined either by theory or experiment, it is logical to conclude that the lack of quantitative agreement between the calculated and experimental particle velocity coefficients in Table II lies in the uncertainty in $\beta_{S}^{\prime}$.

TABLE II. Results of Hugoniot calculations for single-component materials.

\begin{tabular}{lcrcccc}
\hline \hline Material & $\begin{array}{c}u_{1}(\mathrm{~min}) \\
(\mathrm{m} / \mathrm{s})\end{array}$ & $\begin{array}{c}u_{1}(\mathrm{max}) \\
(\mathrm{m} / \mathrm{s})\end{array}$ & $\begin{array}{c}A(\mathrm{Exp}) \\
(\mathrm{km} / \mathrm{s})\end{array}$ & $\begin{array}{c}\text { A ( Calc. }) \\
(\mathrm{km} / \mathrm{s})\end{array}$ & $B(\mathrm{Exp})$ & $B$ (Calc. $)$ \\
\hline $\mathrm{Cu}$ & 350 & 1324 & 3.898 & 3.918 & 1.526 & 1.632 \\
$\mathrm{Zn}$ & 588 & 1237 & 3.040 & 2.989 & 1.539 & 1.765 \\
$\mathrm{Nb}$ & 490 & 1038 & 4.514 & 4.478 & 1.127 & 1.256 \\
$\mathrm{Au}$ & 342 & 680 & 3.058 & 3.016 & 1.568 & 1.759 \\
$\mathrm{Pd}$ & 431 & 1416 & 3.963 & 3.944 & 1.611 & 1.792 \\
$\mathrm{Co}$ & 471 & 946 & 4.709 & 4.664 & 1.381 & 1.544 \\
$\mathrm{~W}$ & 340 & 1156 & 4.008 & 3.975 & 1.278 & 1.361 \\
$\mathrm{Cd}$ & 572 & 1181 & 2.389 & 2.394 & 1.733 & 2.040 \\
$\mathrm{NaCl}$ & 326 & 1746 & 3.488 & 3.361 & 1.309 & 1.658 \\
$\mathrm{Mg}$ & 876 & 1935 & 4.620 & 4.459 & 1.180 & 1.398 \\
$\mathrm{Ag}$ & 471 & 987 & 3.262 & 3.183 & 1.570 & 1.818 \\
$\mathrm{Al}-2024$ & 280 & 1222 & 5.398 & 5.299 & 1.258 & 1.609 \\
$\mathrm{Ni}$ & 475 & 987 & 4.656 & 4.698 & 1.355 & 1.520 \\
$\mathrm{~Pb}$ & 263 & 890 & 2.042 & 1.976 & 1.446 & 1.574 \\
\hline \hline
\end{tabular}


TABLE III. Values of $\beta_{S}^{\prime} \equiv \beta_{S F \text { it }}^{\prime}$ required to fit experimental particle velocity coefficients.

\begin{tabular}{lcccc}
\hline \hline Material & $\beta_{\text {SFII }}^{\prime}$ & $\begin{array}{c}\beta_{\text {SFit }}^{\prime} / \beta_{\text {s }}^{\prime} \\
(\text { Est. })\end{array}$ & $\begin{array}{c}A(\text { Exp. }) \\
(\mathrm{km} / \mathrm{s})\end{array}$ & $\begin{array}{c}A(\mathrm{Calc}) \\
(\mathrm{km} / \mathrm{s})\end{array}$ \\
\hline $\mathrm{Cu}$ & 4.525 & 0.906 & 3.898 & 3.912 \\
$\mathrm{Zn}$ & 4.340 & 0.801 & 3.040 & 2.963 \\
$\mathrm{Nb}$ & 3.025 & 0.852 & 4.514 & 4.476 \\
$\mathrm{Au}$ & 4.450 & 0.844 & 3.058 & 3.010 \\
$\mathrm{Pd}$ & 4.830 & 0.854 & 3.963 & 3.930 \\
$\mathrm{Co}$ & 4.025 & 0.856 & 4.709 & 4.660 \\
$\mathrm{~W}$ & 3.650 & 0.913 & 4.008 & 3.973 \\
$\mathrm{Cd}$ & 5.380 & 0.767 & 2.389 & 2.345 \\
$\mathrm{NaCl}$ & 3.630 & 0.689 & 3.488 & 3.329 \\
$\mathrm{Mg}$ & 3.100 & 0.765 & 4.620 & 4.435 \\
$\mathrm{Ag}$ & 4.550 & 0.804 & 3.262 & 3.166 \\
$\mathrm{Al}-2024$ & 3.555 & 0.711 & 5.398 & 5.293 \\
$\mathrm{Ni}$ & 3.940 & 0.853 & 4.656 & 4.614 \\
$\mathrm{~Pb}$ & 3.750 & 0.862 & 2.042 & 1.968 \\
\hline \hline
\end{tabular}

The data necessary for a comparison between experimental and calculated results for porous materials is available for $\mathrm{Cu}^{18}$ and $\mathrm{Al}-2024 .{ }^{19}$ The Hugoniots of these powders were calculated with the values of $\beta$ 's used to fit the experimental solid Hugoniot particle velocity coefficients, however it should be noted that the results change by no more than $5 \%$ and usually less than $2 \%$ by using the original estimates of $\beta_{S}^{\prime}$ since the elastic energy in a shocked powder is only a small fraction of the total energy. As can be seen in Table IV, the calculated values of both the shock intercept and particle velocity coefficient match the experimental data well with the exception of Al-2024 ( $m=1.27$ ). The discrepancy in this case may be due to inconsistent distensions in the experimental specimens and sparse data. The average absolute difference between the calculated and experimental intercept values is $134 \mathrm{~m} / \mathrm{s}$ and $104 \mathrm{~m} / \mathrm{s}$ with and without the Al-2024, $\mathrm{m}=1.27$, calculation, respectively. The average absolute percentage difference between the experimental and calculated particle velocity coefficients is $3.3 \%$ with the Al-2024, $m=1.27$ calculation and only $1.8 \%$ without it.

The comparison between calculated and experimental Hugoniots in Table IV shows that the theory can quantitatively determine the Hugoniots of a distended single-component material. It is important to emphasize that using the

TABLE IV. Results of Hugoniot calculations for distended single-component materials.

\begin{tabular}{|c|c|c|c|c|c|c|}
\hline Material & $\begin{array}{c}u_{1}(\min ) \\
(\mathrm{m} / \mathrm{s})\end{array}$ & $\begin{array}{c}u_{1}(\max ) \\
(\mathrm{m} / \mathrm{s})\end{array}$ & $\begin{array}{r}A(\text { Exp. }) \\
(\mathrm{km} / \mathrm{s})\end{array}$ & $\begin{array}{c}A \text { (Calc. }) \\
(\mathrm{km} / \mathrm{s})\end{array}$ & $B$ (Exp.) & $B$ (Calc.) \\
\hline \multicolumn{7}{|l|}{$\mathrm{Cu}$} \\
\hline$m=1.13$ & 610 & 1789 & 2.092 & 2.155 & 2.084 & 2.059 \\
\hline$m=1.41$ & 730 & 2018 & 0.718 & 0.805 & 2.208 & 2.166 \\
\hline $\begin{array}{l}m=1.57 \\
\text { Al }-2024\end{array}$ & 769 & 2112 & 0.548 & 0.469 & 2.105 & 2.130 \\
\hline$m=1.27$ & 837 & 2130 & 1.319 & 1.602 & 2.472 & 2.241 \\
\hline$m=1.42$ & 874 & 2130 & 0.704 & 0.898 & 2.304 & 2.249 \\
\hline$m=1.69$ & 953 & 2504 & 0.209 & 0.306 & 2.182 & 2.134 \\
\hline
\end{tabular}

original estimates of $\beta_{s}^{\prime}$ has little effect on the results. This, together with the results discussed for solid materials, shows that the model accurately describes a material's shock response. This implies that the extension of the model to a true mixture should be sufficiently accurate to make certain conclusions about a mixture's Hugoniot since the physical description of a material's shock response is the same in the full two-component theory. In the following section, the Hugoniots of mixtures of the materials in Table I are discussed.

\section{APPLICATION TO MIXTURES AND DISCUSSION}

For the following calculations, averaged properties were determined as follows:

$$
\begin{aligned}
& V_{0 A B}=\sum_{i} x_{i} V_{i}, \\
& (V / \gamma)_{O A B}=\sum_{i} x_{i}(V / \gamma)_{0 i}, \\
& \beta_{O S A B}=V_{O A B}\left(\sum_{i} \frac{x_{i} V_{0 i}}{\beta_{0 S i}}\right)^{-1} \\
& \beta_{O S A B}^{\prime}=\left[\frac{\beta_{O S A B}^{2}}{V_{O A B}} \sum_{i} x_{i} V_{0 i}\left(\frac{1+\beta_{0 S i}^{\prime}}{\beta_{0 S i}^{2}}\right)\right]-1 .
\end{aligned}
$$

Unfortunately, few Hugoniots of well-characterized mixtures have been determined experimentally, however there is sufficient data for a comparison with slightly distended mixtures of sintered $\mathrm{W}$ infiltrated with 24 and 45 wt $\% \mathrm{Cu}^{20}$ The calculated and experimental results for $\mathrm{W}$ 24 wt \% Cu are shown as $C_{S}$ vs $u_{1}$ plots in Fig. 2. The three curves correspond to the Hugoniot calculated assuming averaged properties and the extreme cases in which the $W$ or $\mathrm{Cu}$ absorb no thermal energy. A good fit to data is the calculated Hugoniot where the $\mathrm{W}$ absorbs no thermal energy, which is closer to what one might expect; however, this conclusion is poorly supported since the Hugoniot assuming the opposite extreme also fits the data well and better than the calculated Hugoniot assuming averaged properties.

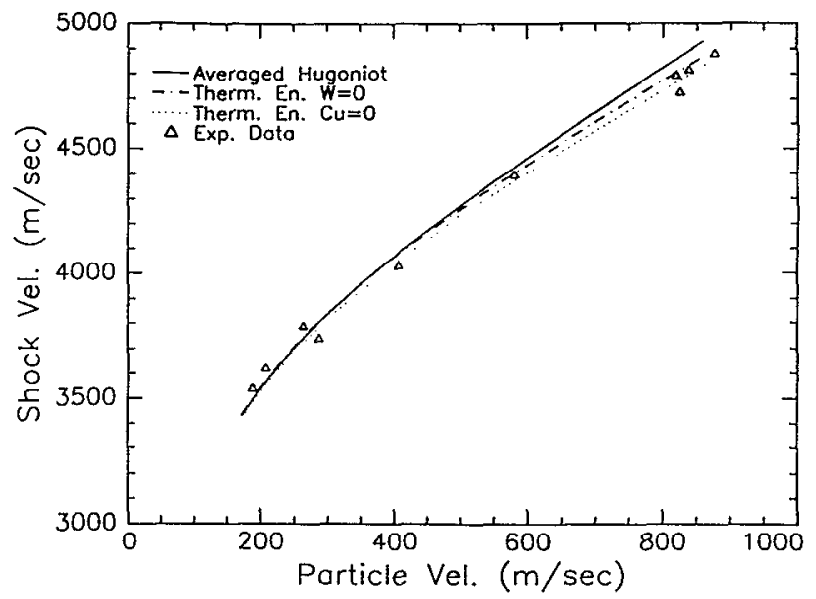

FIG. 2. Calculated and experimental Hugoniots of sintered $W$ infiltrated with $24 \mathrm{wt} \% \mathrm{Cu}$. The distension calculated from the experimental data is 1.014 . 
Interestingly, the calculated Hugoniots are nonlinear at low particle velocities. Although the number of experimental points is small, the data is also nonlinear. All three of the calculated Hugoniots fit the nonlinearity well, however little difference is expected in this range because the thermal energy is smaller at lower particle velocities. McQueen et al. ${ }^{6}$ supposed, by comparison with experimental single-component porous Hugoniots, that the curvature is due to the initial porosity of the samples. Calculations assuming no initial porosity do show the $C_{S}-u_{1}$ relationship to be linear, thereby confirming this conclusion; however, since our model assumes hydrodynamic material behavior and matches the curvature in the experimental data, it can be concluded that the curvature is not the result of material rigidity effects.

The calculated Hugoniot for $\mathrm{W}-45 \mathrm{wt} \% \mathrm{Cu}$ is linear over the range of particle velocities investigated which was higher than in the previous case due to the lack of experimental data at lower particle velocities. The calculated values of $A$ and $B$ are $3.108 \mathrm{~km} / \mathrm{s}$ and 2.014 , respectively, assuming averaged properties, $3.125 \mathrm{~km} / \mathrm{s}$ and 1.917 respectively, assuming the $\mathrm{Cu}$ absorbs no thermal energy, and $3.137 \mathrm{~km} / \mathrm{s}$ and 1.954 , respectively, assuming the $\mathrm{W}$ absorbs no thermal energy. The values of $A$ and $B$ determined from the experimental data over the particle velocities 189 to $878 \mathrm{~m} / \mathrm{s}$ are 3.003 and 2.021 , respectively. It should be noted that one experimental data point was excluded because the density of that particular sample was significantly lower. The calculated values of $A$ and $B$ assuming mass averaged properties are very closed to the experimental values. As with the other W$\mathrm{Cu}$ mixture, the two extreme Hugoniots lie on the same side of the Hugoniot calculated assuming average properties in the $C_{S}-u_{1}$ plane.

The Hugoniots of several other mixtures, listed in Table $V$, have been calculated to investigate the effects of the thermal energy partitioning ratio on the Hugoniots although no experimental data is available for these systems. The weight fraction of the components was taken to be $50 \%$ and the distension to be 1.5. It was assumed that a 304 stainless steel flyer was impacting the sample at velocities from 800 to 2000 $\mathrm{m} / \mathrm{s}$. These systems were chosen because they represent a wide range of possible mixtures, and the shock conditions were chosen because they are typical of shock compaction and shock-initiated reaction experiments. The results are shown in Table $\mathrm{V}$ in the form $C_{S}=A+B^{*} u_{1}$. The results

TABLE V. Results of mixture Hugoniot calculations.

\begin{tabular}{|c|c|c|c|c|c|c|}
\hline \multirow[b]{2}{*}{$\begin{array}{l}\text { System } \\
\text { (!) }\end{array}$} & \multicolumn{2}{|c|}{ Mass-Averaged } & \multicolumn{2}{|c|}{ Therm. En. $1=0$} & \multicolumn{2}{|c|}{ Therm. En. $2=0$} \\
\hline & $\begin{array}{c}A \\
(\mathrm{~km} / \mathrm{s})\end{array}$ & $B$ & $\begin{array}{c}A \\
(\mathrm{~km} / \mathrm{s})\end{array}$ & $B$ & $\begin{array}{c}A \\
(\mathrm{~km} / \mathrm{s})\end{array}$ & $B$ \\
\hline $\mathrm{Cu} / \mathrm{Nb}$ & 0.345 & 2.378 & 0.337 & 2.372 & 0.281 & 2.472 \\
\hline $\mathrm{Mg} / \mathrm{Au}$ & 0.595 & 2.006 & $\cdots$ & $\ldots$ & 0.593 & 2.012 \\
\hline $\mathrm{Cd} / \mathrm{W}$ & 0.393 & 2.331 & 0.399 & 1.991 & 0.299 & 2.385 \\
\hline $\mathrm{Co} / \mathrm{Zn}$ & 0.362 & 2.331 & 0.177 & 2.692 & 0.383 & 2.221 \\
\hline $\mathrm{Cd} / \mathrm{Nb}$ & 0.394 & 2.219 & 0.391 & 2.151 & 0.210 & 2.631 \\
\hline $\mathrm{NaCl} / \mathrm{W}$ & 0.612 & 1.861 & 0.631 & 1.702 & 0.598 & 1.987 \\
\hline
\end{tabular}

for the $\mathrm{Mg} / \mathrm{Au}$ system when the $\mathrm{Mg}$ absorbs no thermal energy have been excluded because the calculated volume of Au was found to be unrealistically high.

One observation drawn from Table $V$ is that unlike the W-Cu system, the Hugoniots in the $C_{S^{-}} u_{1}$ plane straddle the Hugoniot assuming averaged properties, assuming that one component absorbs no thermal energy. Also unlike the W-24 wt $\%$ Cu mixture, the $C_{S}-u_{1}$ relationships are highly linear. The Hugoniots of these mixtures may be nonlinear at lower particle velocities and distensions, however these possibilities were not explored.

Another observation is that in a system where one would expect the thermal energy of one component to be nearly zero, it appears possible to experimentally determine $\xi$ in Eq. (11), however there would be many difficulties in such experiments. First, the calculations have assumed $\xi$ to be constant. It is unlikely that $\xi$ will remain constant over a wide range of shock conditions. More significantly, the resultant effect on the macroscopic shock parameters is relatively small. For example, the largest difference found between an extreme Hugoniot and a Hugoniot assuming averaged properties occurs in the $\mathrm{Cd} / \mathrm{Nb}$ system. This translates into a difference of $4.7 \%, 6.3 \%, 8.8 \%, 2.4 \%$, and $8.8 \%$ in total energy, pressure, shock velocity, particle velocity, and total thermal energy, respectively, at the highest impact velocity. The difference in the total elastic energy is $52 \%$ in this case which may be important in systems with, for example, pressure-induced phase transitions; however, in general this is not as significant as it may seem since the total elastic energy, assuming average properties, is only $7.7 \%$ of the total energy deposited. In the other systems and with lower projectile velocities, the percentage difference in the macroscopic shock parameters is smaller and typically less than $3 \%$. Therefore, to determine $\xi$ a large number of carefully conducted experiments would be required to get statistically significant results, and even then, a small error in the measured shock parameters would result in a large uncertainty in $\xi$.

Since the above calculations are for extremes in thermal energy partitioning and a wide range of possible mixtures has been investigated, it appears that a Hugoniot assuming averaged properties is a valid approximation to the Hugoniot of a mixture under the typical conditions of a shock compaction or shock-initiated reaction experiment even though the thermal energies, and hence temperatures, of the components may differ significantly. Therefore, Eqs. (13)-(16) can be used to determine the averaged properties and Eqs. (1), (2), the flyer pressure-particle velocity relationship and a reduced form of Eq. (8) can be used to calculate the shock parameters for a given flyer velocity. Unfortunately, if a mixture's distension is close or equal to 1, the solid Hugoniots of the components need to be known to determine $\beta_{S}^{\prime}$ until further theoretical or experimental work is done, but in porous mixtures an estimate of $\beta_{s}^{\prime}$ is sufficient since the elastic energy is relatively small.

\section{CONCLUSIONS}

A theory has been presented to determine the Hugoniot of solid and powder two-component mixtures using only 
static pressure data. In developing the model, it was assumed that the pressures and particle velocities of the components were equal while no assumptions were made regarding the relative magnitude of the thermal and elastic energies or temperature equilibrium between the components. The validity of the equal pressure assumption and the fact that temperature equilibrium will not be reached immediately behind the shock front was argued in terms of characteristic equilibration times. The equal particle velocity assumption is based on the fact that no contradictory experimental evidence has been found in our experiments or in the literature.

The model was shown to qualitatively fit the solid $\mathrm{Hu}$ goniots of single-component materials using approximations to $\beta_{S}^{\prime} \equiv\left(\partial \beta_{S} / \partial P\right)_{S} \approx\left(\partial \beta_{S} / \partial P\right)_{T} \approx\left(\partial \beta_{T} / \partial P\right)_{T}$. Furthermore, the experimental data could be fitted by adjusting $\beta$ ' which has not been determined experimentally or theoretically. Using the values of $\beta$ 's fitted to the experimental data, it was shown that the calculated Hugoniots of distended single-component materials fit experimental data well, however approximations to $\beta$ ' are adequate for porous materials since thermal energy terms dominate.

The only mixture without phase changes where experimental data is available over compressions where the model is expected to be valid and where the necessary thermodynamic data can be determined is $\mathrm{W}-\mathrm{Cu}$. The calculated $\mathrm{Hu}-$ goniots for this system were unique in that the Hugoniots assuming either the $\mathrm{W}$ or $\mathrm{Cu}$ absorb no thermal energy lie on the same side of the Hugoniot calculated using averaged properties in the $C_{S}-u_{1}$ plane. Another result for W-Cu is that with 24 wt $\% \mathrm{Cu}$, the $C_{S}-u_{1}$ relationship is nonlinear as is the experimental data. The theory adequately explains the nonlinearity as being due to the initial porosity of the samples.

A series of calculations were then performed on mixtures of materials under typical shock compaction and shock initiated reaction conditions. It was shown that extreme changes in the thermal energy ratio did effect the Hugoniot, however it was argued that the resultant effect on the macroscopic shock parameters is relatively small and would be difficult to determine experimentally even under ideal conditions. Given this result, it can be concluded that a Hugoniot calculated with equations (1), (2), a reduced form of Eq. (8) and the known flyer pressure-particle velocity relationship, and assuming averaged properties using Eqs. (13)(16), is a valid approximation for determining the total energy, pressure, thermal energy, etc. of a shocked mixture.
This indicates that the thermal energy partitioning ratio, which will have an important effect on the shock compaction and shock-initiated reaction processes, will need to be determined by experimental and theoretical means other than by measuring macroscopic shock parameters.

\section{ACKNOWLEDGMENTS}

We would like to thank Andrew Mutz for Fig. 1 and his work on the maraging steel experiment. We would also like to thank Ricardo Schwarz of Los Alamos National Laboratorics for helpful conversations and his thoughtful insight. This work was supported under the National Science Foundation's Materials Processing Initiative Program, Grant No. DMR 8713258. Barry Krueger died on October 29, 1990 as a result of injuries received in a motorcycle accident. His family, friends, and scientific colleagues mourn the loss of a truly gifted individual.

\footnotetext{
'The term initiated is used to imply that the shock wave starts the reaction as opposed to changing the lowest free-energy state of the system [e.g., the shock-induced reaction $\mathrm{C}$ (graphite) $\rightarrow \mathrm{C}$ (diamond) ).

${ }^{2} \mathrm{M}$. B. Boslough, in Proceedings of the Ninth Symposium (International) on Detonation (in press).

'S. S. Batsanov, G. S. Doronin, S. V. Klochdov, and A. I. Teut, in Combus tion, Explosions and Shock Waves (Plenum, New York, 1986), Vol. 22, p. 765.

${ }^{4}$ R. A. Graham, B. Morison, Y. Horie, E. L. Venturini, M. B. Boslough, M. Carr, and D. L. Williamson, in Shock Waves in Condensed Matter, edited by Y. M. Gupta (Plenum, New York, 1986), p. 693.

${ }^{5}$ N. N. Thadhani, M. J. Costello. I. Song, S. Work, and R. A. Graham, in Proceedings of the TMS Symposia on Solid State Powder Processing, Indianapolis, October $1-4,1989$ (to be published).

'R. G. McQueen, S. P. Marsh, J. N. Taylor, J. N. Fritz, and W. J. Carter, in High Velocity Impact Phenomena, edited by R. Kinslow (Academic, New York, 1970), p. 293.

${ }^{7}$ G. E. Duvall and S. M. Taylor, Jr., J. Compos. Mater. 5, 130 (1971).

${ }^{8}$ R. L. Williamson, R. N. Wright, G. E. Korth, and B. H. Rabin, J. Appl. Phys. 66, 1826 (1989).

${ }^{9}$ R. B. Schwarz, P. Kasiraj, and T. Vreeland, Jr, in Metallurgical Applications of Shock Waves and High-Strain-Rate Phenomena, edited by L. E. Murr, K. P. Staudhammer, and M. A. Meyers (Dekker, New York, 1986), p. 313

"'M. B. Boslough and R. A. Graham. Chem. Phys. Lett. 121, 446 (1985).

"M. B. Boslough, J. Chem. Phys. 92, 1839 (1990).

${ }^{12}$ F. D. Murnaghan, Proc. Nat. Acad. Sci. 30, 244 (1944),

${ }^{13}$ O. L. Anderson, J. Phys. Chem. Solids. 27, 547 (1966).

${ }^{14}$ V. N. Zharkov and V. A. Kalinin, Equations of State for Solids at High Pressures and Temperatures (Consultants Bureau, New York, 1971).

15J. S. Dugdale and D. K. C. MacDonald, Phys. Rev. 89, 832 (1953).

${ }^{\text {in }}$ K. Oh and P. Persson, J. Appl. Phys., scheduled for 15 Nov. 90.

${ }^{17}$ P. S. Marsh, Ed., LASL Shock Hugoniot Data (University of California Press, Berkeley, 1980).

${ }^{18}$ Reference 17, pp. 63-65.

${ }^{14}$ Reference 17, pp. 175-179.

${ }^{20}$ Reference 17, pp. 523-525.
} 